\title{
General Principles for the Nonequilibrium Relaxation of Populations in Quantum Materials
}

\author{
A. F. Kemper, ${ }^{1, *}$ O. Abdurazakov, ${ }^{1}$ and J. K. Freericks ${ }^{2}$ \\ ${ }^{1}$ Department of Physics, North Carolina State University, Raleigh, North Carolina 27695, USA \\ ${ }^{2}$ Department of Physics, Georgetown University, Washington, DC 20057, USA
}

(Received 22 August 2017; revised manuscript received 20 April 2018; published 15 October 2018)

\begin{abstract}
We examine the problem of how excited populations of electrons relax after they have been excited by a pump. We include three of the most important relaxation processes: (i) impurity scattering, (ii) Coulomb scattering, and (iii) electron-phonon scattering. The relaxation of an excited population of electrons is one of the most fundamental processes measured in pump-probe experiments, but its interpretation remains under debate. We show how several common assumptions about nonequilibrium relaxation that are pervasive in the field may not hold under quite general conditions. The analysis shows that nonequilibrium relaxation is more complex than previously thought, but it yields to recently developed theoretical methods in nonequilibrium theory. In this work, we show how one can use many-body theory to properly interpret and analyze these complex systems. We focus much of the discussion on implications of these results for experiment.
\end{abstract}

DOI: 10.1103/PhysRevX.8.041009

\section{INTRODUCTION}

In the analysis of nonequilibrium theory and experiments, it is common to lean on intuition developed from equilibrium physics and from linear response, even in circumstances where the system is driven far from equilibrium. The experiments are typically performed with a pump-probe setup, where the pump drives the system out of equilibrium and out of the domain where our equilibrium intuition applies. The measurement is made with a wide variety of probes in a similar variety of contexts, and they have been the subject of rapid development. Theory has also improved beyond the Boltzmann equation and effective temperature approaches, with exact methods being developed in one dimension (via density matrix renormalization group [1]) and in infinite dimensions (via nonequilibrium dynamical mean-field theory [2,3]). While much work has been accomplished in both cases, there are a number of assumptions that underlie a significant fraction of the analysis in the field which do not always hold. We describe four of these assumptions in detail here and fully consider three of them, leaving the resolution of the fourth to later work.

The four assumptions are as follows: (i) many-body systems must relax after excitation, (ii) the self-energy

\footnotetext{
*akemper@ncsu.edu
}

Published by the American Physical Society under the terms of the Creative Commons Attribution 4.0 International license. Further distribution of this work must maintain attribution to the author(s) and the published article's title, journal citation, and DOI.
Subject Areas: Condensed Matter Physics,

Optics, Strongly Correlated Materials

governs the relaxation rate, (iii) the time domain allows one to separate the relaxation rates from different scattering processes, and (iv) when electrons are coupled to phonons, they rapidly scatter amongst themselves to create hot thermal electrons that subsequently relax with the phonons until they both reach a common final temperature.

The reasons why each of these assumptions may be readily violated follow from a rather straightforward analysis:

(i) Isolated electronic systems cannot relax back to the state before the pump (equilibrium) because their total energy (given by their initial energy plus the energy imparted by the pump) is conserved. Hence, while they can rearrange the energy amongst their constituents, in many cases this does not evolve to a thermal distribution. Recent work on the eigenstate thermalization hypothesis says that systems without additional conserved quantum numbers do evolve to a thermal distribution, while those with additional conserved quantities will evolve to generalized Gibbs distributions which maintain the additional conservation laws [4]. In addition, pumped systems sometimes are excited into metastable states or novel nonequilibrium phases, which may relax in unknown ways (and not to the prepump state) because these phases are not present in thermal equilibrium [5]. We show that pure impurity scattering does not cause relaxation to thermal equilibrium while pure Coulomb scattering should. When electrons are attached to a phonon bath with electron-phonon coupling, they can relax in a way that removes energy from the electronic system and eventually 
returns the system to the temperature of the phonon bath.

(ii) When the many-body system does relax, early work in many-body physics suggested that it is the imaginary part of the self-energy that governs the relaxation rate [6-9], similar to the situation in equilibrium $[10,11]$. While one can show this suggestion holds for inelastic scattering processes in nonequilibrium when both the coupling and pump are weak, it is violated by stronger interactions and higher pump fluence [12]. An even more significant violation has been reported in experiments [13,14], but a full explanation of this effect does not exist yet. In this work, we point towards a resolution of this conundrum.

(iii) Matthiessen's rule [15] governs the relaxation of most systems in linear response. It says that one adds the relaxation rates for different scattering processes to yield one net relaxation rate (similar to how resistors add as reciprocals when in a parallel circuit). As with the electrical circuit analogy, one should see only the net relaxation rate in the time dynamics. Yet, in numerous experiments, one does see a separation of relaxation rates for excited populations. We show this naturally occurs in nonequilibrium relaxation and leads to violations of Matthiessen's rule.

(iv) Finally, the hot-electron model, which has been employed in many different contexts, can be easily shown to be incorrect. An equation of motion for the population shows that whenever one has a fluctuation-dissipation-like relationship $\left[A^{<}(\omega) \propto\right.$ $f(\omega) \operatorname{Im} A^{R}(\omega)$ ], where the lesser quantities (propagators and self-energies) are given by the imaginary parts of retarded quantities multiplied by some distribution function (it need not even be an equilibrium distribution), then the population no longer relaxes with time. In fact, one can easily show that it is the deviations from the hot-electron model that govern the nonequilibrium relaxation [16,17]. We summarize the argument for why this must occur. Given the wide application of hot-electron models to analyzing pump-probe experiments, this failure is our most important result. We discuss later under what circumstances hot-electron model analyses make sense (as good approximations) and under what situations they fail.

Quite generally, these assumptions break down due to the fundamental difference between measurement time and quasiparticle lifetime. By nature, equilibrium measurements (through linewidth, for example) observe the quasiparticle lifetime that arises from Fourier transformation along relative time; these observations access the dephasing or decay of the correlation functions. On the other hand, nonequilibrium measurements see the energy transfer between various subsystems rather than this dephasing.

Some of these assumptions have been previously treated theoretically within Boltzmann equation approaches $[18,19]$, whose authors find similar results regarding the limitations of the hot-electron model and the separation of timescales. However, Boltzmann equation approaches by nature do not capture the emergence of the history kernel, and thus provide no description of the retarded nature of the interactions and frequency dependence of the electron Green's functions. Nevertheless, these works find discrepancies between the inherent dephasing time of a Fermi gas and the population dynamics after the pump [18], and the critical appearance of bottlenecks [19], which we further elucidate here.

To demonstrate that these assumptions may be readily violated for simple systems, we compare and contrast relaxation from impurities, coupling of electrons to phonons, electron-electron scattering, and various combinations. Although these are relatively simple scattering processes that are well understood in equilibrium, we show that their characteristics in the time domain are not simple extensions from equilibrium. Furthermore, we use them as counterexamples to show that even in simple cases the assumptions may be violated. We show how some of the examples behave differently because they cannot fully relax, and how their temporal dynamics shows clear violations of Matthiessen's rule, with the origin arising from details for how energy is transferred from electrons to phonons. We also describe the implications of this work for the interpretation of experiments, in particular, emphasizing the different characteristic behaviors of the different scattering processes.

\section{MODEL AND METHOD}

We investigate the aforementioned scattering channels: electron-impurity, electron-phonon, and electron-electron (Coulomb) scattering through the Hubbard-Holstein model with local interactions

$$
\begin{aligned}
\mathcal{H}= & \sum_{\mathbf{k}, \sigma} \epsilon(\mathbf{k}) c_{\mathbf{k}, \sigma}^{\dagger} c_{\mathbf{k}, \sigma}+\bar{U} \sum_{i} c_{i \uparrow}^{\dagger} c_{i \uparrow} c_{i \downarrow}^{\dagger} c_{i \downarrow}+\sum_{i, \sigma} \bar{V}_{i} c_{i, \sigma}^{\dagger} c_{i, \sigma} \\
& +\Omega \sum_{i} b_{i}^{\dagger} b_{i}+g \sum_{\mathbf{k}, \mathbf{q}, \sigma} c_{\mathbf{k}+\mathbf{q}, \sigma}^{\dagger} c_{\mathbf{k}, \sigma}\left(b_{\mathbf{q}}+b_{-\mathbf{q}}^{\dagger}\right)
\end{aligned}
$$

where the individual terms represent the kinetic energy of electrons with a dispersion $\epsilon(\mathbf{k})$, the energy of Einstein phonons with a frequency $\Omega$, and a coupling between them whose strength is given by $g$. Here, $c_{\alpha}^{\dagger}\left(c_{\alpha}\right)$ are the standard creation (annihilation) operators for an electron in state $\alpha$; similarly, $b_{\alpha}^{\dagger}\left(b_{\alpha}\right)$ creates (annihilates) a phonon in state $\alpha$. The $\bar{U}$ and $\bar{V}_{i}$ terms represent the on-site Coulomb interaction and random impurity scattering, respectively. We study a square lattice dispersion with nearest- and nextnearest-neighbor hopping $\left(t_{\mathrm{NN}}\right.$ and $\left.t_{\mathrm{NNN}}\right)$, 


$$
\begin{aligned}
\epsilon(\mathbf{k})= & -2 t_{\mathrm{NN}}\left[\cos \left(k_{x}\right)+\cos \left(k_{y}\right)\right] \\
& +4 t_{\mathrm{NNN}} \cos \left(k_{x}\right) \cos \left(k_{y}\right)-\mu,
\end{aligned}
$$

where $\mu$ is the chemical potential. We use the convention that $\hbar=c=e=1$, which makes inverse energy the unit of time. We choose $t_{\mathrm{NN}}=0.25 \mathrm{eV}, t_{\mathrm{NNN}}=0.075 \mathrm{eV}$, and $\mu=-0.255 \mathrm{eV}$, and use an inverse temperature $\beta=100 / \mathrm{eV}$.

The self-energies for the three different scattering mechanisms are shown in the Feynman diagrams in Fig. 1. The electron-phonon interaction is treated at the self-consistent Born level [Fig. 1(a)], with the self-energy given by

$$
\Sigma_{\mathrm{el-ph}}^{\mathcal{C}}\left(t, t^{\prime}\right)=i g^{2} G_{\mathrm{loc}}^{\mathcal{C}}\left(t, t^{\prime}\right) D_{0}^{\mathcal{C}}\left(t, t^{\prime}\right) .
$$

Here, $D_{0}^{\mathcal{C}}\left(t, t^{\prime}\right)$ is the noninteracting phonon propagator [11], and $G_{\mathrm{loc}}^{\mathcal{C}}\left(t, t^{\prime}\right)=N_{\mathbf{k}}^{-1} \sum_{\mathbf{k}} G_{\mathbf{k}}^{\mathcal{C}}\left(t, t^{\prime}\right)$ is the local Green's function. The superscript $\mathcal{C}$ denotes that the quantity lives on the two-time Keldysh contour [20]. In this formulation, we assume the phonons have an infinite heat capacity, so their temperature does not rise due to coupling with the excited electrons. Hence, they maintain the same phonon propagator for all times. This approximation is valid for situations where the amount of energy put into the system is not large enough to cause a notable change in the phonon dynamics [21].

Similarly, the impurity scattering self-energy after averaging [Fig. 1(b)] is given by

$$
\Sigma_{\mathrm{el-imp}}^{\mathcal{C}}\left(t, t^{\prime}\right)=V^{2} G_{\mathrm{loc}}^{\mathcal{C}}\left(t, t^{\prime}\right) .
$$

$V$ is obtained from impurity averaging as $V^{2}=n_{\text {imp }} \bar{V}^{2}$, where $n_{\text {imp }}$ is the density of impurities, and we neglect the momentum dependence of the scattering matrix element.

The electron-electron interactions are included at the level of self-consistent conserving second-order perturbation theory [Fig. 1(c)] via

$$
\Sigma_{\mathrm{el}-\mathrm{el}}^{\mathcal{C}}\left(t, t^{\prime}\right)=\bar{U}^{2} G_{\mathrm{loc}}^{\mathcal{C}}\left(t, t^{\prime}\right) G_{\mathrm{loc}}^{\mathcal{C}}\left(t, t^{\prime}\right) G_{\mathrm{loc}}^{\mathcal{C}}\left(t^{\prime}, t\right),
$$

which is equivalent to a dynamical mean-field approximation with a conserving perturbative impurity solver. This

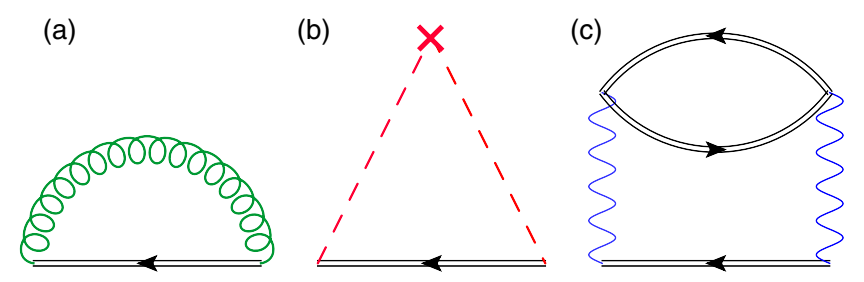

FIG. 1. Self-energies used in this study. (a) Electron-phonon self-energy in the Migdal approximation. (b) Impurity self-energy in the Born approximation. (c) Second-order electron-electron self-energy. All of these are treated self-consistently. approximation neglects the momentum dependence of the two-particle bubble, which increases the phase space for electron-electron scattering but should produce accurate results as long as the interaction is not too large. For local interactions, the Hartree-Fock terms only shift the chemical potential.

Note that we do not include any mixed self-energy diagrams between the different scattering channels. This is not a requirement, but it allows for a clear differentiation between the different scattering mechanisms themselves, and it is the lowest-order contribution in perturbation theory for each scattering channel.

In all three cases, there exists a sum rule for the interactions at this level of perturbation theory. As was discussed previously [12,22], the frequency-integrated electron-phonon interactions obey a sum rule. The impurity and Coulomb-scattering self-energies also obey similar sum rules. They are as follows:

$$
\begin{aligned}
\operatorname{Im} \sum_{\text {el-ph }}^{R}(t, t) & =-g^{2}\left[\left\langle x^{2}(t)\right\rangle-\langle x(t)\rangle^{2}\right] \\
& =-g^{2}\left[2 n_{B}(\Omega / T)+1\right], \\
\operatorname{Im} \Sigma_{\text {el-imp }}^{R}(t, t) & =-V^{2}, \\
\operatorname{Im} \Sigma_{\text {el-el }}^{R}(t, t) & =-\bar{U}^{2} n(1-n) \equiv-U^{2},
\end{aligned}
$$

where $n=\left\langle n_{\uparrow}\right\rangle=\left\langle n_{\downarrow}\right\rangle$ is the electron density per spin, and $n_{B}(\Omega)$ is the Bose occupation of the phonon mode $\Omega$. These identities are true at all times and hold individually even when all three types of interactions are present. To put the parameter strength for the various interactions on an equal footing, we define an interaction strength $U^{2}=$ $\bar{U}^{2} n(1-n)$ which takes into account the dependence of the electron-electron scattering on the electron density.

The equations of motion for the Green's functions are solved on the contour by using the methods described in

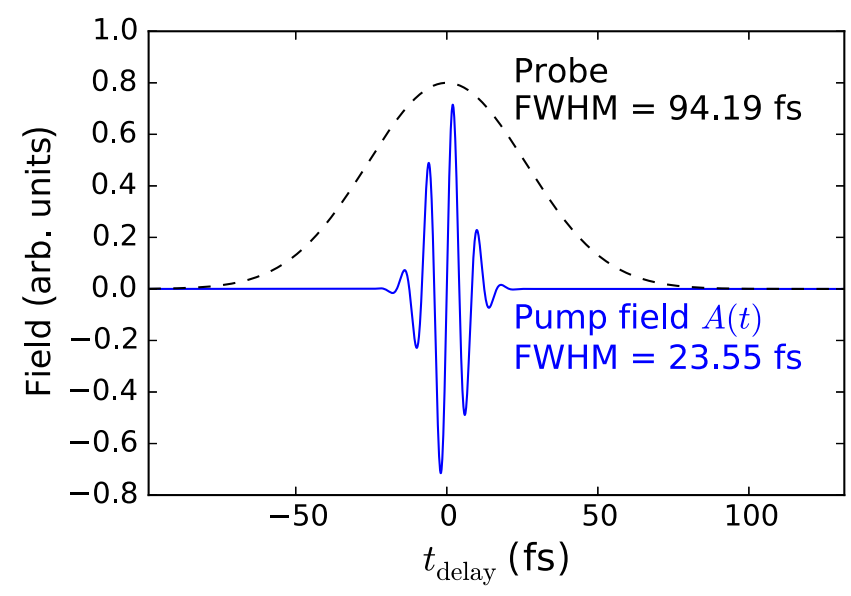

FIG. 2. Pump vector potential $A(t)$ and probe profiles used in this work. The probe profile is the envelope function for the probe pulse. 
Ref. [23]. The field is included via the Peierls substitution [24] $\mathbf{k}(t)=\mathbf{k}-\mathbf{A}(t)$, where the vector potential $\mathbf{A}(t)$ is treated in the Hamiltonian gauge. We use a pump of the form $A(t)=A_{\max } \exp \left(-t^{2} / 2 \sigma^{2}\right) \sin (\omega t)$ in the diagonal (11) direction with $\omega=0.5 \mathrm{eV}$. The field is illustrated in Fig. 2. The full width at half maximum of the field is approximately $24 \mathrm{fs}$, while the width of the probe is approximately 94 fs (see below). The timescales for the fields are chosen for computational reasons, as are the interaction strengths and related timescales. However, since we are focused on the postpump dynamics, the time- and energy scales in the pump do not have an imprint on the physics discussed below; rather, the pump is only a mechanism to make an excitation.

\section{A. Observables}

The single-particle angle-resolved photoemission spectral functions are obtained from the Green's functions via [25]

$$
\begin{aligned}
& P\left(\mathbf{k}, \omega, t_{\text {delay }}\right) \\
& \quad=\operatorname{Im} \int d t_{1} d t_{2} p\left(t_{1}, t_{2}, t_{\text {delay }}\right) e^{i \omega\left(t_{1}-t_{2}\right)} G_{\mathbf{k}\left(t_{1}, t_{2}\right)}^{<}\left(t_{1}, t_{2}\right),
\end{aligned}
$$

where $p\left(t_{1}, t_{2}, t_{\text {delay }}\right)$ is a two-dimensional normalized Gaussian with isotropic width $\sigma_{p}=\sigma$ centered at $t_{1}=t_{2}=t_{\text {delay }}$ :

$$
\begin{aligned}
& p\left(t_{1}, t_{2}, t_{\text {delay }}\right) \\
& \quad=\frac{1}{2 \pi \sigma^{2}} \exp \left(-\frac{\left(t_{1}-t_{\text {delay }}\right)^{2}+\left(t_{2}-t_{\text {delay }}\right)^{2}}{2 \sigma^{2}}\right) .
\end{aligned}
$$

The Gaussian probe profile along $t_{\text {delay }}$ is shown in Fig. 2 . The shift in momentum due to the Peierls substitution has to be corrected to obtain gauge-invariant spectra through a time-dependent shift of the momentum [26,27] via

$$
\overline{\mathbf{k}}\left(t, t^{\prime}\right)=\mathbf{k}+\frac{1}{t-t^{\prime}} \int_{t^{\prime}}^{t} \mathbf{A}(\bar{t}) d \bar{t} .
$$

Below, we also consider the amount of excited electrons by evaluating the electron density above $k_{F}$ :

$$
n\left(k>k_{F}\right)=-i \sum_{k>k_{F}} G_{\mathbf{k}}^{<}\left(t, t^{\prime}=t\right) .
$$

\section{RESULTS}

To set the stage for the discussion, we outline the oftenused conceptual framework underlying the assumptions. First, in equilibrium, one typically identifies the quasiparticle lifetime with the self-energy through

$$
\frac{1}{\tau(\nu)}=-2 \operatorname{Im} \Sigma^{R}(\nu),
$$

where we combine the quasiparticle quantum numbers into a single index $\nu$. This relation was suggested to hold in the time domain within certain limits with $\tau$ as the population decay rate rather than the single-particle lifetime [6-9], but it was experimentally shown not to hold in at least a few cases $[13,14]$. One of the consequences of this line of thought is that since the self-energy is always present, relaxation must always occur.

Next, in equilibrium, the scattering rates for each of the relevant processes encoded in the self-energy add linearly for weak coupling. Based on this concept, time- and angle-resolved photoemission spectroscopy (tr-ARPES) experiments are commonly analyzed by considering the contributions of various scattering processes to the population decay rate based on estimates from equilibrium experiments. Although the addition of the scattering rates should give rise to a single exponential decay rate, this is often not observed - a multiexponential type of behavior is seen instead. This agrees with the mode of thinking that the processes in the time domain should separate according to their fundamental timescales. Based on converting the characteristic energy scales into timescales and ranking them, the strong interaction (often electron-electron) is thought to occur first, followed by the weaker interactions (e.g., electron-phonon scattering).

Below, we show that these concepts are erroneous. The population decay rate is not simply connected to the selfenergy, and the scattering rates do not always separate. Rather, the population decay rate is set by a difference between effective distributions of the populations and the self-energy, and any separation of scattering rates arises from energy bottlenecks.

We begin by considering the effects of individual electron-phonon, electron-impurity, and electron-electron (Coulomb) scattering in a pump-probe setup. Figure 3 shows snapshots of the tr-ARPES spectra for the three individual interactions under consideration (videos of the three processes are available in the Supplemental Material). To make a proper comparison, the interaction strengths, as defined through the sum rules in Eq. (6d), are kept constant. The equilibrium spectra at $t_{\text {delay }}=-86 \mathrm{fs}$ show the typical hallmarks of a band of electrons interacting with phonons, impurities, and internally through Coulomb scattering.

The system with phonon scattering shows a kink in the spectra at the phonon frequency together with a sharpening of the spectra within the phonon window (defined as $|\omega|<\Omega$ ). This window plays a key role in determining the population dynamics at low energies due to phase-space restrictions for phonon emission [9,12,28].

The system with impurity scattering has a more or less homogenous scattering rate proportional to the density of states. Coulomb scattering, within second-order perturbation theory, gives rise to a self-energy (and, thus, a scattering rate) that is given by $\operatorname{Im} \Sigma \sim-\left(\omega^{2}+\pi^{2} T^{2}\right)$. The temperature here is sufficiently low that the $\omega^{2}$ term 


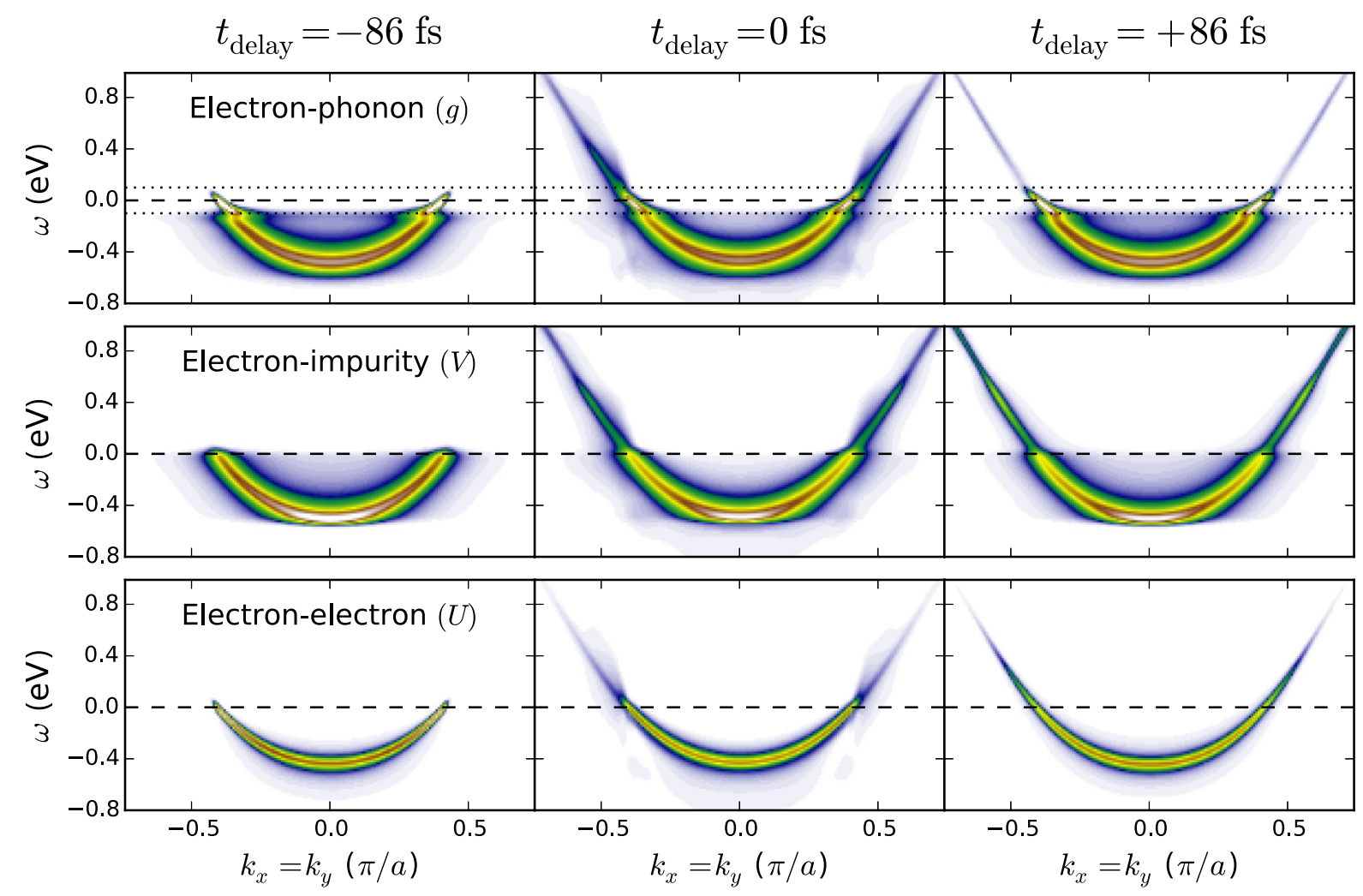

FIG. 3. False-color images of time-resolved single-particle spectral functions at the start of (left), during (middle), and after (right) the pump for the individual electron-phonon, electron-impurity, and electron-electron interactions. Here, the interaction strengths are $g^{2}=V^{2}=U^{2}=0.02 \mathrm{eV}$. The dotted line indicates the phonon frequency $\Omega= \pm 0.1 \mathrm{eV}$.

dominates, which is reflected in the spectra in the increase in the linewidth at high energies.

The spectra at $t_{\text {delay }}=0 \mathrm{fs}$ are at the point where the pump and probe overlap. In all three cases, there is excited spectral weight above the Fermi level; there are some qualitative distinctions between the spectra that originate from the particular interactions (e.g., the weakening of the kink in the phonon-scattering case), but the excitation is present in all cases. This scenario is also similar to what one would envision from the hot-electron approach. But, if the electronic system fully relaxes to a hot temperature, we show below that it never subsequently relaxes to a lower temperature.

\section{A. tr-ARPES for individual scattering channels}

The final snapshot at $t_{\text {delay }}=+86 \mathrm{fs}$ is taken long after the pump. At this point, the system with phonon scattering has partially completed its return to equilibrium, as has been studied in some detail previously $[8,9,12]$. This is clearly reflected in the partial disappearance of the excited spectral weight above the Fermi level (see, also, Fig. 4). The systems where only impurity or Coulomb scattering are present are in stark contrast to the former case-after the pump, they essentially do not return to the equilibrium state before the pump. However, although these two share the characteristic of a new steady state after the pump, the appearance of the spectra is quite different. For impurity scattering, there is essentially no change in the spectra immediately after the pump, which agrees with previous analytic work [16] showing a steady state once momentum equilibration is achieved. This is expected because the final state should be a generalized Gibbs [4] state which will not look thermal. On the other hand, the system with Coulomb

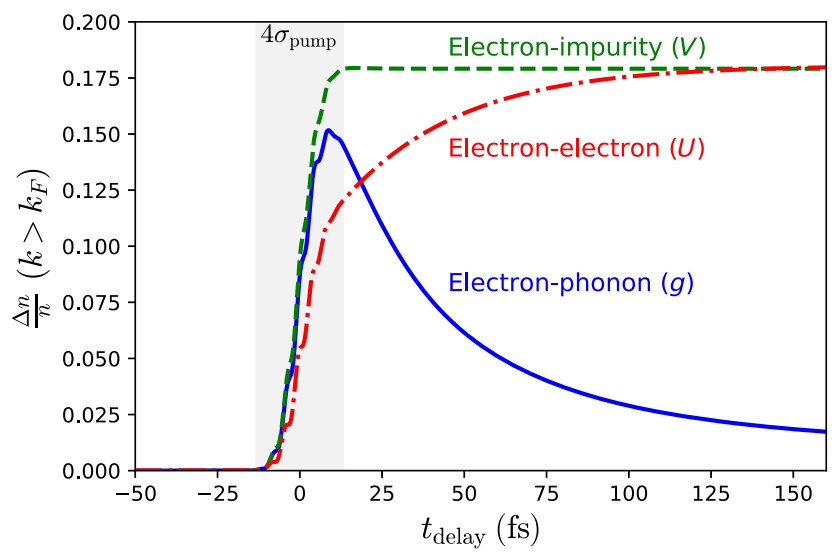

FIG. 4. Quasiparticle weight above the Fermi level for the individual electron-phonon, electron-impurity, and electron-electron interactions. The shaded region indicates $\pm 2 \sigma$ of the pump field. 
scattering exhibits some short-time dynamics and quickly settles into the state seen in the figure. The short-time dynamics is caused by internal thermalization of the electrons, and the steady state is an elevated temperature state, as expected by the eigenstate thermalization hypothesis.

Figure 4 illustrates the difference between the three scattering processes through the excited population above the Fermi level [see Eq. (10)]. The electron-phonon interactions behave as expected, with an excitation followed by a return to equilibrium. On the other hand, the impurity and Coulomb-scattering cases end up in a state with a sizable persistent increase in the population above the Fermi level, although the Coulomb-scattering system takes some time to get there as it scatters electrons from below $E_{F}$.

These observations immediately demonstrate a violation of two of the assumptions: Many-body systems do not always relax to the equilibrium state before the pump even long after the pulse ends [assumption (i)], nor does the selfenergy directly govern the relaxation rate [assumption (ii)].

\section{B. Impurity and phonon scattering}

Figures 3 and 4 show that the different interactions exhibit qualitatively different temporal dynamics beyond the quantitative aspect that is expected due to their different nature. Here, we show the error in assumption (ii), the selfenergy governs the relaxation rate, by considering the combination of impurity and phonon scattering in some detail. Figure 5 shows the equilibrium effect of increasing impurity scattering on top of a constant phonon-scattering strength. The impurity scattering self-energy is proportional to the density of states, which adds (according to Matthiessen's rule) to the existing phonon self-energy, increasing the overall single-particle scattering rate and filling in the phonon window. This increase is reflected in the spectra, in which the phonon kink can be seen when $V^{2}=0$, but it is rapidly obscured as impurity scattering increases. This can be further analyzed by fitting the spectral momentum distribution cuts (MDCs), which yield the dispersion $\epsilon(\mathbf{k})$ and MDC linewidths. The dispersion shows the kink gradually disappearing, and the MDC widths show a monotonic increase as well as the disappearance of the step in the single-particle scattering rate. At the highest impurity scattering strength, where $V^{2}=2.5 \times g^{2}$, the phonon window and kink are effectively gone.

If the self-energy were to control the relaxation rate of the population, then the relaxation rate should increase as the impurity scattering increases. Figure 6(a) plots the decay rates as a function of quasiparticle energy $\epsilon(\mathbf{k})$, as the impurity scattering is increased from $V^{2}=0$. The figure clearly shows that the dramatic effect on the equilibrium measures (MDC linewidth, self-energy) are wholly absent in the nonequilibrium population decay rates.
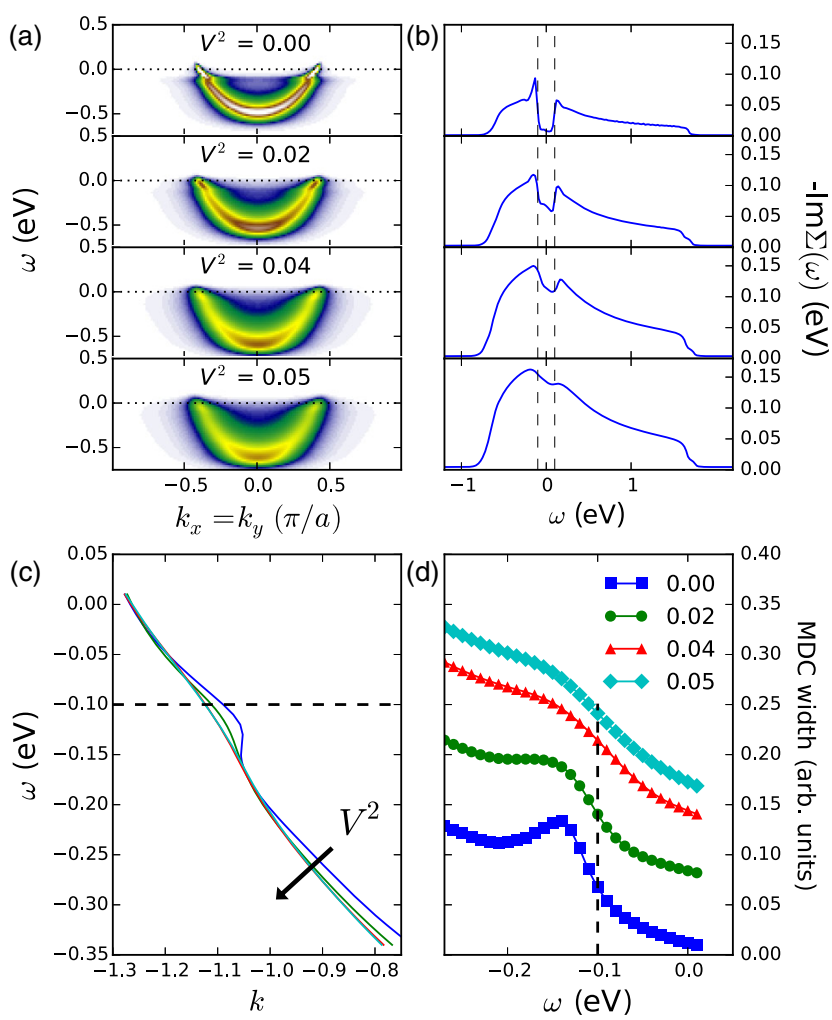

FIG. 5. Effect of combined electron-impurity and electronphonon scattering in equilibrium. (a),(b) Equilibrium ARPES spectra and self-energies $[-\operatorname{Im} \Sigma(\omega)]$ for various impurity scattering strengths $V$. The phonon-scattering strength is kept constant at $g^{2}=0.02 \mathrm{eV}$. (c),(d) Extracted dispersion $\epsilon(\mathbf{k})$ and MDC widths from the ARPES spectra.

This demonstrates a violation of assumption (ii): The self-energy governs the relaxation rate by contradiction; there are substantial changes in the self-energy due to the presence and strength of the impurity scattering that are not observed in the nonequilibrium population dynamics where the changes are minor. As we see, aside from the weakcoupling limit of pure electron-phonon scattering [9], this result turns out to be the generic behavior of nonequilibrium systems with a range of different scattering mechanisms.

For this mixed-interaction case, it appears that the electron-phonon scattering is the dominant mechanism in the return to equilibrium. We have previously argued that this is because phonons can draw energy out of the system [28]. Indeed, an isolated electronic system must conserve its total energy, and, hence, it cannot dissipate its excess energy. Impurities scatter quasiparticles throughout the Brillouin zone but do so while conserving energy and, thus, cannot drive the system back to equilibrium with a lower temperature. In this case, assumption (iii), which says Matthiessen's rule is violated, does hold, but in an overstated way; since the impurities are inefficient in relaxing the electrons, we see much too mild of a dependence of the scattering rate on the impurity scattering 
strength. Hence, we do not see a separation of the scattering into an impurity-based scattering and an electron-phononbased scattering.

This is further highlighted in Fig. 6(b), which shows the population above the Fermi level evaluated according to Eq. (10). If assumption (iii) were to hold, then there should be a double exponential decay with a time constant for each process. To illustrate that this is not the case, we show that the curves can be reasonably fit using a single exponential (although some deviations are visible). Thus, the timescales clearly do not separate simply based on the presence of multiple scattering channels with different coupling constants.

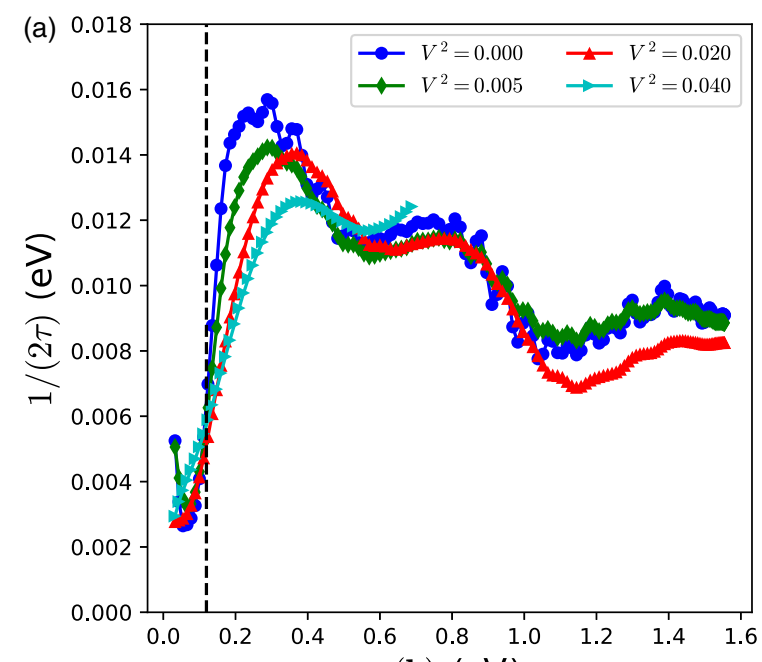

$\epsilon(\mathbf{k})(\mathrm{eV})$

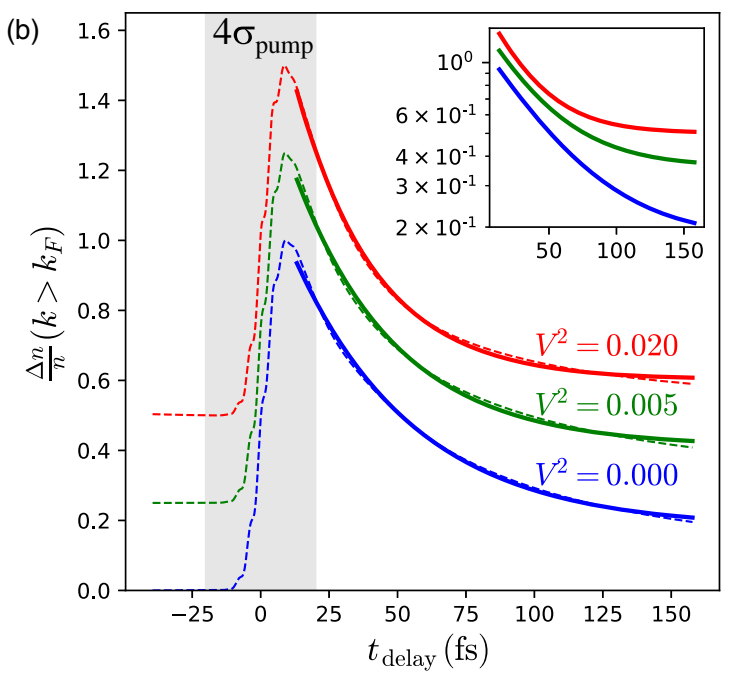

FIG. 6. (a) Population decay rates after excitation showing a weak dependence on the impurity scattering rate $V$ (values for $V^{2}$ are in $\mathrm{eV}^{2}$ ). (b) Normalized quasiparticle weight above the Fermi level for phonon and impurity scattering (offset for clarity). The solid lines are single exponential fits to the region where the solid line is shown, which should not work if timescales separate. The shaded region indicates $\pm 2 \sigma$ of the pump field. Inset: Semilogarithmic plot of the same data.
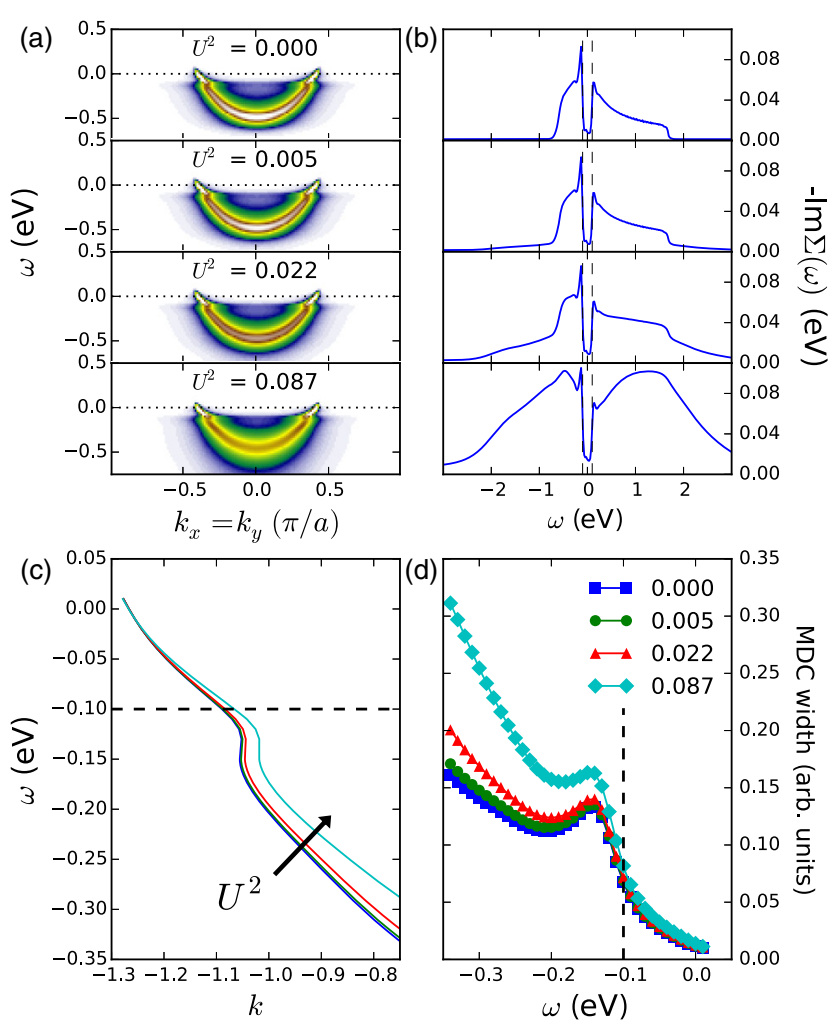

FIG. 7. Effect of combined electron-electron and electronphonon scattering in equilibrium. (a),(b) Equilibrium ARPES spectra and self-energies $[-\operatorname{Im} \Sigma(\omega)]$ for various Coulombscattering strengths $U$. The phonon-scattering strength is kept constant at $g^{2}=0.02 \mathrm{eV}$. (c),(d) Extracted dispersion $\epsilon(\mathbf{k})$ and MDC widths from the ARPES spectra.

\section{Coulomb and phonon scattering}

Coulomb, or electron-electron scattering, is critically different from impurity scattering in that it is inelastic; it can redistribute energy among quasiparticles (although the total energy in the quasiparticle system is conserved). This can be seen in Fig. 3 as a difference between the spectra at $t_{\text {delay }}=0 \mathrm{fs}$ and $t_{\text {delay }}=86 \mathrm{fs}$. Shortly after the pump, the electrons rearrange to form a steady state that is different from equilibrium (although it is thermal). When phonon scattering is also present, a complex interplay between the two scattering channels emerges, which we use to demonstrate a violation of assumption (iii), the time domain allows one to separate the relaxation rates from different scattering processes. In addition, violation of assumption (ii) is illustrated once again.

We consider the dynamics of a system subject to both Coulomb and phonon scattering. Figure 7 shows the same analysis as that performed for the impurity scattering: the effects of increasing Coulomb scattering on the equilibrium spectra. Here, since the Coulomb-scattering self-energy is proportional to $\omega^{2}$ at low temperatures, the main effect on the self-energy occurs at high energies, and the phonon window remains intact [Fig. 7(b)]. These changes in the 
self-energy are reflected in the spectra [Fig. 7(a)], which continue to show the phonon kink but gain an increased linewidth at higher energies. This is further confirmed quantitatively through the MDC fits in both the dispersion and linewidth analysis [Figs. 7(c) and 7(d)].

Turning now to the population dynamics in the time domain, we observe a very different situation from impurity scattering, as is clear from Fig. 8(a). There is a notable effect with increasing Coulomb scattering, but the effect is not the linear increase one would expect from Matthiessen's rule. While a linear increase is observed in
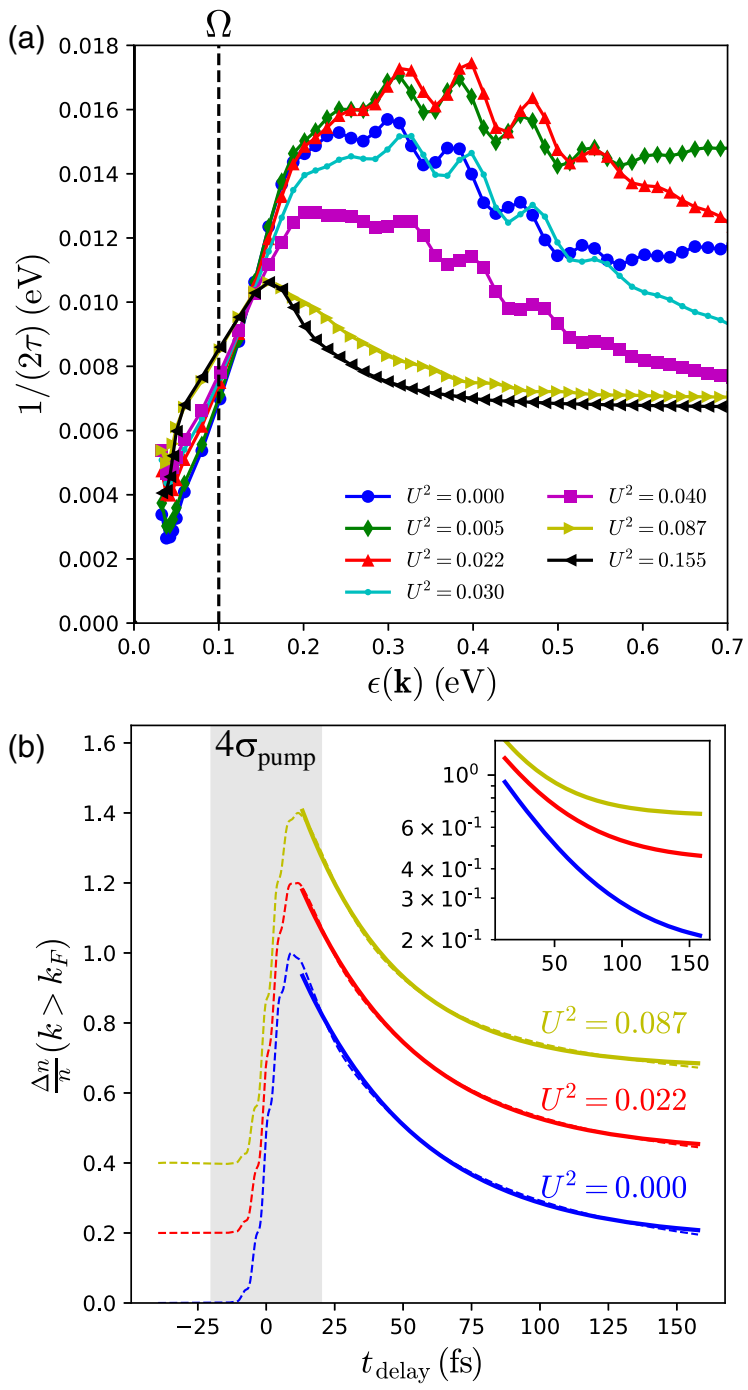

FIG. 8. (a) Population decay rates after excitation showing a nonmonotonic dependence on the Coulomb-scattering strength $U$ (values of $U^{2}$ are in $\mathrm{eV}^{2}$ ). Outside the phonon window, after an initial increase, the decay rates decrease. Some of the data are reproduced from Ref. [28]. (b) Quasiparticle weight above the Fermi level for phonon and Coulomb scattering (offset for clarity). The solid lines are single exponential fits to the region where the solid line is shown, which should not work if timescales separate. The shaded region indicates $\pm 2 \sigma$ of the pump field. Inset: Semilogarithmic plot of the same data. the self-energies, which increase with the addition of Coulomb scattering [cf. Fig. 7(b)], the population decay rates initially show a modest increase, which subsequently reverses with a stronger Coulomb-scattering strength, in particular at high energies above the phonon window. The phonon window itself remains essentially visible, although the size of the step in $1 / 2 \tau$ decreases. We can clearly see a violation of Matthiessen's rule and that the self-energy does not directly govern the relaxation rate. Furthermore, just as for the case of combined phonon and impurity scattering, the population decay in time [Fig. 8(b)] does not show two distinct relaxation times with a double exponential form nor is there a marked increase in the decay rate with increasing $U$. It is dominated by the electron-phonon relaxation time, with a small increase in the rate due to Coulomb rearrangement of the quasiparticles enabling slightly more efficient phonon emission. The decay is controlled by the phonons because they provide the effective path and, thus, a bottleneck of sorts for the electrons to lose their excess energy.

\section{DISCUSSION}

\section{A. What does Mathiessen's rule suggest?}

Is it instructive to reiterate the conclusions one would draw from a simple Mathiessen's rule analysis. If the decay rates are to be proportional to the excited density to arrive at an exponential decay, we may expect the populations to obey a simple differential equation:

$$
\frac{d n}{d t}=-\frac{1}{\tau_{1}} n-\frac{1}{\tau_{2}} n+\cdots=-\frac{1}{\tau_{\text {eff }}} n .
$$

Regardless of the number of channels, one would arrive at a single exponential decay rate. Moving beyond that, to arrive at a double exponential (with decay constants $\gamma_{1}$ and $\gamma_{2}$ ), one would need a second-order differential equation of the form

$$
\frac{d^{2} n}{d t^{2}}=-\left(\gamma_{1}+\gamma_{2}\right) \frac{d n}{d t}-\gamma_{1} \gamma_{2} n .
$$

The exact equations of motion may, in limited cases, be reduced to Eqs. (12) or (13) but generally do not fit either of these [16]. Hence, one should not expect these simple behaviors to occur. In no circumstances is there any simple explanation for multiple relaxation times in terms of the equations of motion for the population.

\section{B. Origin of the breakdown of the assumptions}

The contrast between Figs. 5 and 7 (equilibrium) and Figs. 3, 4, 6, and 8 (nonequilibrium) are the central results that demonstrate violation of the assumptions:

(1) Assumption (i) - many-body states relax after excitation-is contradicted by the simple examples of 
single-channel scattering through either impurities or Coulomb scattering, neither of which decay but both of which are interacting many-body systems.

(2) Assumption (ii) - the self-energy governs the relaxation rate-is contradicted by a comparison between the equilibrium self-energy (Figs. 5 and 7 ) and the population dynamics (Figs. 3, 4, 6, 8). The selfenergies monotonically increase as the second scattering channel is added, whereas the population decay rates show entirely different behavior. This explains the experimental results which show large differences between equilibrium linewidths and nonequilibrium decay rates $[13,14]$.

(3) Assumption (iii) - the time domain allows one to separate the relaxation rates from different scattering processes-is contradicted by Figs. 6 and 8. As evidenced from Fig. 6, impurity scattering (in the Born approximation) can be removed entirely from the consideration of energy-resolved decay rates since it plays no role in the return to equilibrium. Electron-electron scattering plays a more intricate role when it is added to phonon scattering (cf. Fig. 8), but it does not appear that electron-electron scattering always happens first, followed by phonon scattering, even though the energy scales are quite different. In either case, going to the time domain does not separate out the timescales for scattering. In fact, in situations where Matthiessen's rule holds, there is only one effective relaxation time determined by the different scattering mechanisms. In situations where Matthiessen's rule is violated and the different timescales do emerge in nonequilbrium, separation of timescales occurs due to other reasons such as bottlenecks associated with the transfer of energy from the electrons to the phonons. Indeed, such a bottleneck is the only clear way one can obtain such a separation.

Given that these assumptions are readily violated, what is the reason for their violation? The answer partly lies in the distinction between relative time dynamics $\left(t_{\text {rel }}=t-t^{\prime}\right)$ and average (measurement) time dynamics $\left[t_{\text {ave }}=\right.$ $\left.\frac{1}{2}\left(t+t^{\prime}\right)\right]$, also known as the delay time $t_{\text {delay. }}$. In equilibrium, the quasiparticle lifetime arises from the decay of the quasiparticle propagator in relative time; through Fourier transformation, this decay gives rise to a linewidth. Once one goes into the measurement time domain, the $t_{\text {ave }}$ time axis becomes relevant. As a function of average time, the dynamics along relative time for the Green's function $G$ and the self-energy $\Sigma$ may be different. It is precisely this difference that gives rise to the (measurement) average time dependence as we discuss in the next section.

This fundamental difference between the quasiparticle lifetime and the measurement time dependence is the primary source of the breakdown of the assumptions. Equilibrium measurements by nature measure in the
Fourier domain that comes from the transform along $t_{\text {rel }}$, e.g., the equilibrium quantities (self-energy, MDC linewidth) illustrated in Figs. 5 and 7. Out of equilibrium, these quantities may change as functions of measurement time in addition to the changes in the quasiparticle distribution. However, the dynamics along the average time direction is controlled by energy transfer rather than the dephasing of the correlation functions along $t_{\text {rel }}$, and time-domain experiments principally access the former aspect of the physics.

\section{What determines the population decay rate?}

Given that the self-energy alone does not directly govern the decay rate, the question arises: "What does?" We can gain some insight into this question by making a connection to the equilibrium situation. For a system at long enough times after the pump such that $t_{\text {delay }} \equiv t_{\text {ave }}-$ $t_{\text {pump }} \gg \tau_{C}$ (where $\tau_{C}$ is the characteristic dephasing time for the Green's functions and self-energies), the equation of motion for the populations reads

$$
\begin{gathered}
\frac{d n_{\mathbf{k}}\left(t_{\mathrm{ave}}\right)}{d t_{\mathrm{ave}}} \equiv-\left.i \frac{d G_{\mathbf{k}}^{<}(t, t)}{d t}\right|_{t \rightarrow t_{\mathrm{ave}}} \\
=\int_{-\infty}^{t} d \bar{t}\left\{\Sigma^{R}(t, \bar{t}) G_{\mathbf{k}}^{<}(\bar{t}, t)+\Sigma^{<}(t, \bar{t}) G_{\mathbf{k}}^{A}(\bar{t}, t)\right. \\
\left.-G_{\mathbf{k}}^{<}(t, \bar{t}) \Sigma^{A}(\bar{t}, t)-G_{\mathbf{k}}^{R}(t, \bar{t}) \Sigma^{<}(\bar{t}, t)\right\} .
\end{gathered}
$$

The long-time assumption is required for there to be no explicit dependence on the vector potential (which has decayed to zero by this time).

If the dynamics along $t_{\text {ave }}$ is sufficiently slow that the $G$ and $\Sigma$ are constants as a function of $t_{\text {ave }}$ on the scale of $\tau_{C}$, we can perform a Wigner transformation $\left(t, t^{\prime}\right) \rightarrow\left(t_{\text {ave }}, t_{\text {rel }}\right)$ and Fourier transform along $t_{\text {rel }}$ to find

$$
\begin{aligned}
\frac{d n_{\mathbf{k}}\left(t_{\mathrm{ave}}\right)}{d t_{\mathrm{ave}}}= & \int_{-\infty}^{\infty} d \omega\left\{2 i \operatorname{Im}\left[\Sigma^{R}\left(t_{\mathrm{ave}}, \omega\right)\right] G_{\mathbf{k}}^{<}\left(t_{\mathrm{ave}}, \omega\right)\right. \\
& \left.-2 i \operatorname{Im}\left[G_{\mathbf{k}}^{R}\left(t_{\mathrm{ave}}, \omega\right)\right] \Sigma^{<}\left(t_{\mathrm{ave}}, \omega\right)\right\} .
\end{aligned}
$$

We are now in a position to define the electron and interaction distribution functions for the Green's function via

$$
f^{G}\left(t_{\mathrm{ave}}, \omega\right) \equiv i G_{\mathbf{k}}^{<}\left(t_{\mathrm{ave}}, \omega\right) / 2 \operatorname{Im} G_{\mathbf{k}}^{R}\left(t_{\mathrm{ave}}, \omega\right),
$$

and similar for the self-energy $\Sigma\left(t_{\text {ave }}, \omega\right)$. This definition is motivated by the fluctuation-dissipation theorem, which holds identically in equilibrium with the distribution functions $f^{G}\left(t_{\text {ave }}, \omega\right)=f^{\Sigma}\left(t_{\text {ave }}, \omega\right)=n_{F}(\omega, T)$, where $n_{F}(\omega, T)$ is the Fermi distribution function at the system temperature $T$. 
Using these distribution functions, we can obtain

$$
\begin{aligned}
\frac{d n_{\mathbf{k}}\left(t_{\text {ave }}\right)}{d t_{\text {ave }}}= & |2 i|^{2} \int_{-\infty}^{\infty} d \omega \operatorname{Im}\left[\Sigma^{R}\left(t_{\text {ave }}, \omega\right)\right] \operatorname{Im}\left[G_{\mathbf{k}}^{R}\left(t_{\text {ave }}, \omega\right)\right] \\
& \times\left[f^{G}\left(t_{\text {ave }}, \omega\right)-f^{\Sigma}\left(t_{\text {ave }}, \omega\right)\right] .
\end{aligned}
$$

For sufficiently well-defined quasiparticles, this can be further simplified to

$$
\begin{aligned}
\frac{d n_{\mathbf{k}}\left(t_{\mathrm{ave}}\right)}{d t_{\mathrm{ave}}}= & |2 i|^{2} \operatorname{Im}\left[\Sigma^{R}\left(t_{\mathrm{ave}}, \epsilon_{\mathbf{k}}\right)\right] \operatorname{Im}\left[G_{\mathbf{k}}^{R}\left(t_{\mathrm{ave}}, \epsilon_{\mathbf{k}}\right)\right] \\
& \times\left[f^{G}\left(t_{\mathrm{ave}}, \epsilon_{\mathbf{k}}\right)-f^{\Sigma}\left(t_{\mathrm{ave}}, \epsilon_{\mathbf{k}}\right)\right] .
\end{aligned}
$$

The decay rate of the population at a momentum $\mathbf{k}$ is thus obtained from the difference in distributions for the self-energy $\Sigma$ and the populations themselves (through $G_{\mathbf{k}}^{<}$). From this relation, we can conclude the following:

(1) We can immediately observe the necessity for allowing different distribution functions for $G$ and
$\Sigma$ : If they were equal, then no dynamics would occur because the right-hand side would be identically 0 .

(2) Although the self-energy is involved, the return to equilibrium is determined by a combination of $\operatorname{Im} \Sigma^{R}\left(t_{\mathrm{ave}}, \omega\right)$ and the distributions.

(3) If we use the hot-electron model, where the $f^{G} / f^{\Sigma}$ functions are thermal functions with the same temperature, then there is no dynamics at all except if a different "temperature" is assumed for $\Sigma$ and $G$. In this sense, it is the deviations from the hotelectron model that arise when $f^{G} \neq f^{\Sigma}$ that control the dynamics.

(4) For (elastic) electron-impurity scattering within the self-consistent Born approximation, the right-hand side of the equation vanishes identically [16]. This is generically the case for any self-energy which has the form

$$
\Sigma^{\mathcal{C}} \propto \sum_{\mathbf{k}} G_{\mathbf{k}}^{\mathcal{C}}
$$

\section{Phonon}
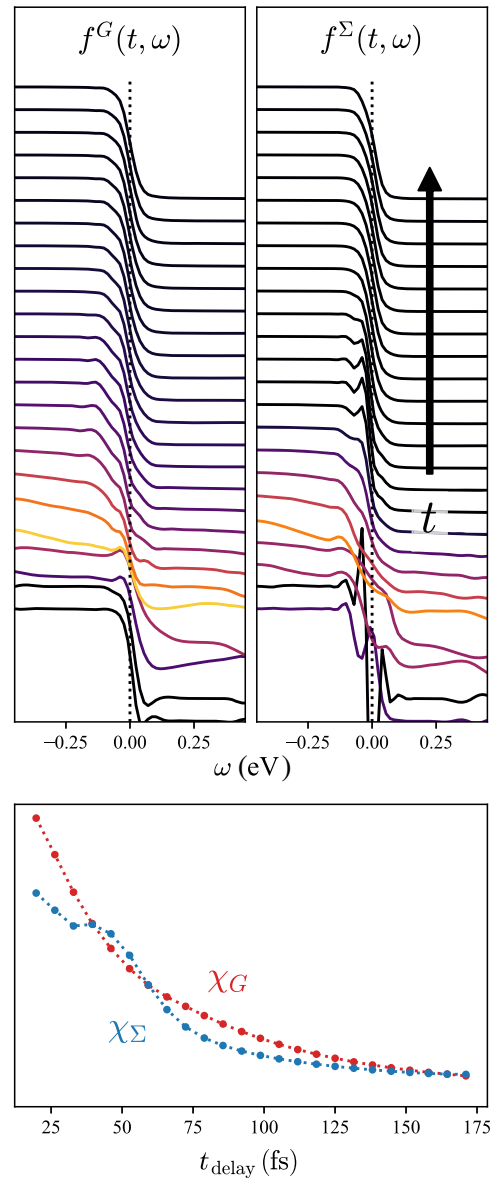

Phonon + Coulomb
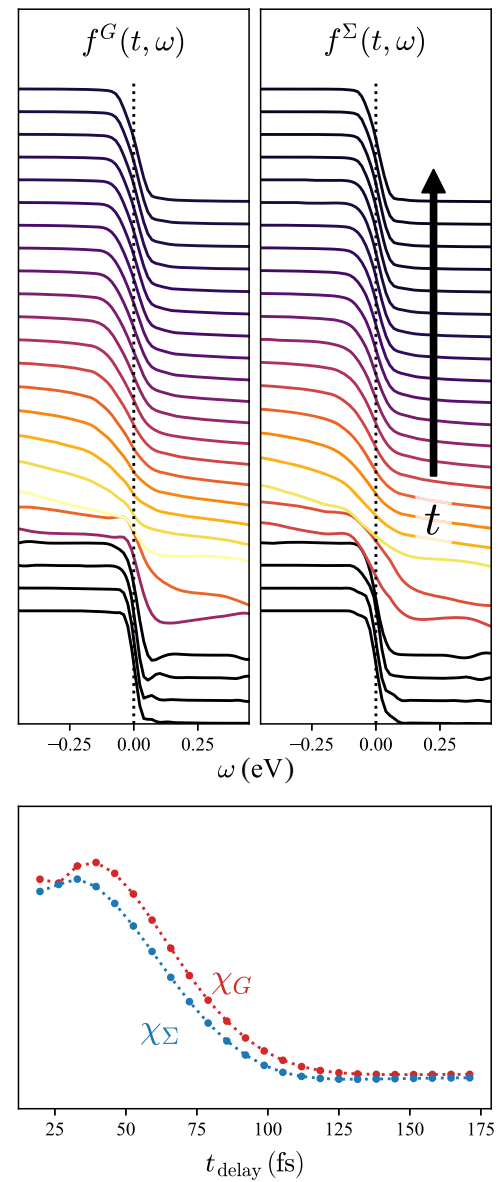

\section{Coulomb}
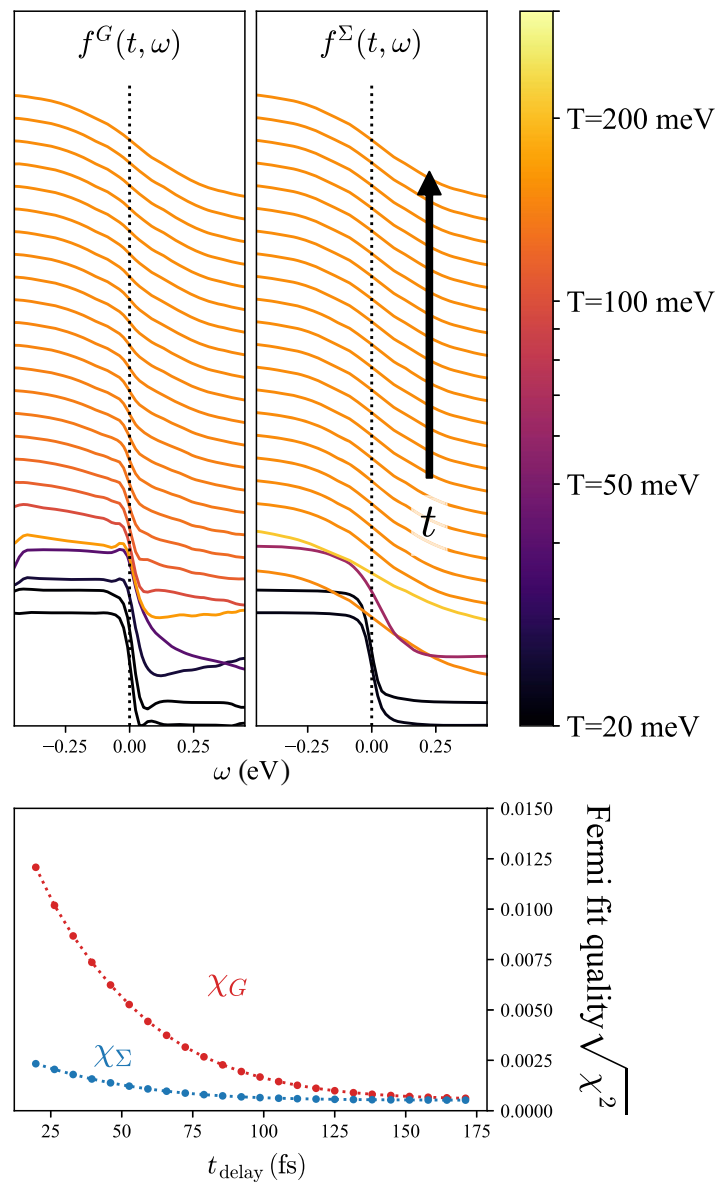

FIG. 9. Top: Population and interaction distribution functions $f^{G}\left(\omega, t_{\text {ave }}\right)$ and $f^{\Sigma}\left(\omega, t_{\text {ave }}\right)$ for three of the cases considered. The line color indicates the temperature obtained from a Fermi function fit. Bottom: $\sqrt{\chi^{2}}$ corresponding to the Fermi function fits for the three cases above it. 
where the sum may be over the entire or a restricted Brillouin zone. This is due to the fact that the distributions $f^{G}$ and $f^{\Sigma}$ must be identical in this case.

The dynamical variable that controls the population dynamics is thus not just the population itself (encoded through $f^{G}$ ) but rather the difference between the distributions for the Green's function $G$ and the self-energy $\Sigma$. These distributions $f^{G}\left(t_{\text {ave }}, \omega\right)$ and $f^{\Sigma}\left(t_{\text {ave }}, \omega\right)$ depend on both frequency and average (measurement) time.

We perform the change to Wigner coordinates followed by the Fourier transformation of the full two-time numerical results. Figure 9 shows the distribution functions $f^{G} / f^{\Sigma}$ for phonon scattering, Coulomb scattering, and the combination as a function of measurement time and frequency. The unusual shapes at early times arise due to the Fourier transform overlapping with the pump for early measurement times. As time progresses, the sharp step in the distributions (whose origin is the sharp step in the Fermi function at low temperatures) smoothes out in both the population and interaction distributions. The subsequent dynamics then depends on the particular scattering mechanism. In the case of simple phonon scattering, the distributions quickly return to a low temperature and have sharp features due to the phase-space restrictions on the phonon scattering. On the other hand, pure Coulomb scattering produces smooth, high-temperature-like distributions quickly after the pump. The mixed case interpolates between the two-it does return to the low-temperature (prepump) state but does so in a smoother way than pure phonon scattering does.

As noted above, the dynamics of the populations is determined by the difference of the population and interaction distribution functions. To illustrate this and to make a connection to the concept of quasitemperature, we perform Fermi function fits of the distribution functions $f^{G} / f^{\Sigma}$ and plot the resulting quasitemperatures $T_{G} / T_{\Sigma}$ as a function of time (Fig. 10). Note that the Fermi function fitting is not perfect and that there is some deviation from the functional form. This is illustrated in Fig. 9, where the fit quality (through a $\chi^{2}$ measure) is shown. The approach to perfect Fermi-Dirac form varies from case to case (and is best for pure Coulomb scattering). In particular, when phonon scattering is present, a finite offset to the long-time population remains for long times due to phonon window effects. The situation with pure impurity scattering is sufficiently far from Fermi functions whose fits were not even attempted.

In all cases, the dynamics in Fig. 10 shows that the two fitted quasitemperatures approach each other at long times. The case of pure Coulomb scattering is qualitatively different because of its approach to a high-temperature state, as we discuss above. The cases where some phonon scattering is present all approach the phonon bath temperature, although the pathway varies somewhat depending on the types of
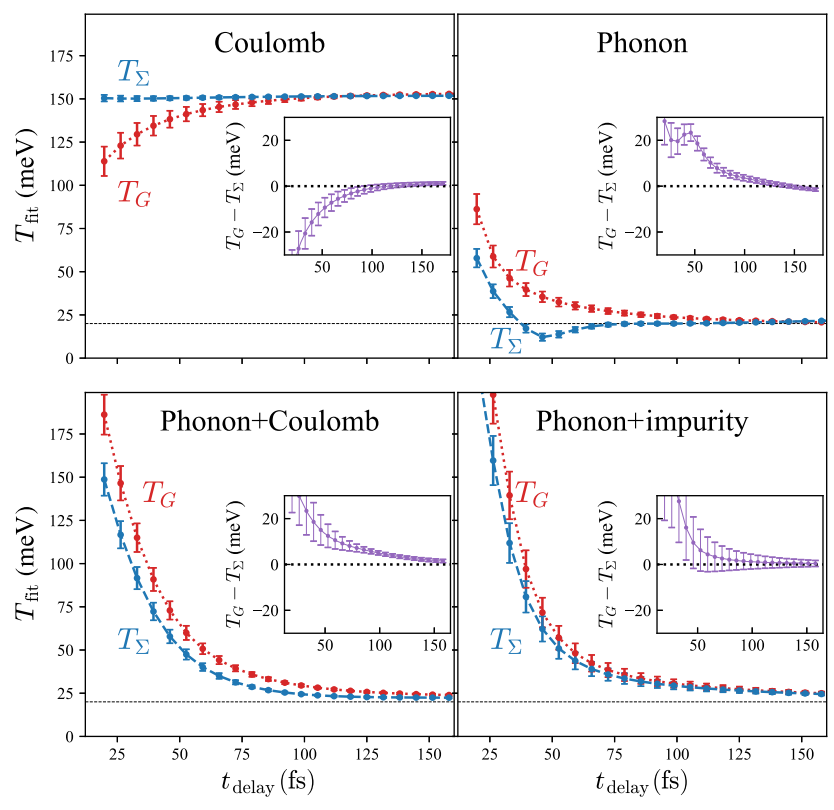

FIG. 10. Effective temperatures extracted from fits to FermiDirac functions for the Green's function (populations) and selfenergy. The horizontal line indicates the equilibrium temperature before the pump. The insets show the difference between the population and interaction effective temperatures.

interactions. Since the dynamical variable is not the population distribution function $f^{G}$ or the extracted temperature $T_{G}$ but rather the difference $\left(f^{G}-f^{\Sigma}\right)$ or $\left(T_{G}-T_{\Sigma}\right)$, we show the temperature difference in the insets. The difference approaches zero in all cases but with varying dynamics and even with apparently time-dependent rates.

In view of these results, it should be noted that the hotelectron model is often applied successfully to experimental data [29]. This is not surprising - the hot-electron model typically produces a decaying exponential-like curve, possibly with a final state that is at some elevated temperature. This behavior certainly mirrors some of the experimental results, although it has been previously pointed out that the hot-electron model arises only from the limiting case where electron-electron collisions are faster than the electron-phonon ones [19]. Theoretical work also tends towards thermal solutions because of the typical use of Boltzmann equation approaches which have thermal states as their fixed point and do not allow for separate distributions in the population and interactions unless the phonon distribution is explicitly included [18,19].

Although this is an exact proof that the assumptions for the hot-electron model do not hold in any many-body system, we cannot easily quantify how close a hot-electron analysis may be to the exact result. It may, in fact, be an accurate approximation, in particular if the long-time dynamics in the self-energy is slow. However, it is never exact. 


\section{Implications for experiments}

Although the dynamics are determined by a difference between the two distributions $f^{G}$ and $f^{\Sigma}$, typical experiments such as tr-ARPES have ready access to observe only one of these: $f^{G}$, as measured by the populations. As such, it is not immediately possible to determine the entire righthand side of the equation of motion which would be necessary to extract any information about the self-energy $\Sigma$. The question is then: Given that only part of the equation $\left(f^{G}\right)$ can be observed, what does its analysis show?

The answer may be found in Figs. 6 and 8, where such an analysis is performed. In discussing these figures and results, we note that although the self-energy may have multiple components, the energy-dependent rates are mainly determined by the phonon scattering. As shown previously, the contribution from the impurity self-energy typically cancels [16]. To understand the remainder after the removal of the impurity scattering within the context of the distribution functions, we can rewrite the equation of motion in terms of the change in the distributions $\delta f^{G} / \delta f^{\Sigma}$ :

$$
\begin{aligned}
\frac{d\left[\delta n_{\mathbf{k}}\left(t_{\text {ave }}\right)\right]}{d t_{\text {ave }}}= & |2 i|^{2} \int_{-\infty}^{\infty} d \omega \operatorname{Im}\left[\Sigma^{R}\left(t_{\text {ave }}, \omega\right)\right] \operatorname{Im}\left[G_{\mathbf{k}}^{R}\left(t_{\text {ave }}, \omega\right)\right] \\
& \times\left[\delta f^{G}\left(t_{\text {ave }}, \omega\right)-\delta f^{\Sigma}\left(t_{\text {ave }}, \omega\right)\right],
\end{aligned}
$$

where we subtract a term proportional to $f_{\text {eq }}^{G}(\omega)-$ $f_{\mathrm{eq}}^{\Sigma}(\omega)=0$. This modification to the equation defines

$$
\begin{gathered}
\delta f^{G}\left(t_{\mathrm{ave}}, \omega\right) \equiv f^{G}\left(t_{\mathrm{ave}}, \omega\right)-f_{\mathrm{eq}}^{G}(\omega), \\
\delta f^{\Sigma}\left(t_{\mathrm{ave}}, \omega\right) \equiv f^{\Sigma}\left(t_{\mathrm{ave}}, \omega\right)-f_{\mathrm{eq}}^{\Sigma}(\omega),
\end{gathered}
$$

since the equilibrium distributions are equal for $G$ and $\Sigma$. For simple electron-phonon scattering, at high energies and for weak pumping, the change in the self-energy $\Sigma\left(t_{\text {ave }}, \omega\right)$ from equilibrium is typically not large, and we may approximate $\delta f^{\Sigma}\left(t_{\text {ave }}, \omega\right) \approx 0$. We can combine the remaining factors through the definition of $f^{G}$, and for quasiparticles that are sufficiently well defined, find

$$
\begin{aligned}
\frac{d\left[\delta n_{\mathbf{k}}\left(t_{\mathrm{ave}}\right)\right]}{d t_{\mathrm{ave}}} & =2 i \int_{-\infty}^{\infty} d \omega \operatorname{Im}\left[\Sigma^{R}\left(t_{\mathrm{ave}}, \omega\right)\right] \delta G_{\mathbf{k}}^{<}\left(t_{\mathrm{ave}}, \omega\right) \\
& \approx 2 i \operatorname{Im}\left[\Sigma^{R}\left(t_{\mathrm{ave}}, \epsilon_{\mathbf{k}}\right)\right] \int_{-\infty}^{\infty} d \omega \delta G_{\mathbf{k}}^{<}\left(t_{\mathrm{ave}}, \omega\right) \\
& =-2 \operatorname{Im}\left[\Sigma^{R}\left(t_{\mathrm{ave}}, \epsilon_{\mathbf{k}}\right)\right] \delta n_{\mathbf{k}}\left(t_{\mathrm{ave}}\right) .
\end{aligned}
$$

Equation (25) follows from the well-defined quasiparticle approximation because $G_{\mathbf{k}}^{<}(\omega)$ is sharply peaked at $\omega=\epsilon_{\mathbf{k}}$.

This expression suggests that within this approximation, a relatively simple exponential decay may occur, with $2 \operatorname{Im} \Sigma^{R}\left(t_{\text {ave }}, \epsilon_{\mathbf{k}}\right)$ as the time constant. Note that this result is different from the hot-electron model because we explicitly assume a difference between the distribution functions for $G$ and $\Sigma$.

This analysis may be extended to any situation where the change in the populations is in a sense larger than that in the interactions. Within this limit, the simple picture may also be applied. The full solution requires the relaxation of the assumptions made in this section, and the result is the modification of the simple decay picture with the complications discussed in this paper and elsewhere $[12,28]$. This point is clearly seen in Fig. 10, where $T_{\Sigma}\left(t_{\text {ave }}\right)$ is not negligible. In addition, inclusion of the phonon degrees of freedom will further make changes to the equations of motion that will strongly modify $f^{\Sigma}$, in particular at later times. We reserve a study of this phenomenon for future work. However, we do know that in the long-time limit, the first correction for the case where the time evolution is slow results in a large number of difference terms that do not seem to have any simple form; no one term dominates over another [17].

\section{When are the assumptions valid?}

As we discuss throughout this manuscript, there are situations where the assumptions-although not generally true-may nevertheless hold. These may include the following:

(i) Assumption (i) that many-body systems must relax after excitation is often not at issue-empirically, all photoexcited systems eventually return to their prepump equilibrium state. Our point here is that for the return to equilibrium, there must be an energy reservoir and energy transfer-in contrast to a linewidth which is always present when the self-energy is nonzero.

(ii) Assumption (ii) that the self-energy governs the decay is true in limited cases. In particular, when the interactions are dominated by a single-scattering process between the excited electrons and some bath (e.g., phonons), the decay is determined by the selfenergy [28]. However, going beyond this simple situation breaks down this assumption.

(iii) Assumption (iii) that relaxation rates separate in the time domain holds when each process is associated with a bottleneck of some sort. If both scattering processes can happen at the same time, no separation occurs.

(iv) Assumption (iv) that electrons thermalize among themselves never holds in the strict sense where both of the distributions are determined by the same thermal distribution function. However, functionally it appears that at least the distribution function for the populations $f^{G}$ is often reasonably thermal [29]. As we show, however, $f^{\Sigma}$ cannot be the same thermal distribution or no dynamics will occur. 


\section{CONCLUSIONS AND OUTLOOK}

In this work, we show by contradiction that several assumptions which are pervasive in nonequilibrium experiments may be violated in certain circumstances. To whit, these assumptions are as follows: (i) many-body systems relax after excitation; (ii) the self-energy governs the relaxation rate; (iii) the time domain allows one to separate the relaxation rates from different scattering processes; (iv) electrons rapidly scatter amongst themselves to create hot thermal electrons before subsequently scattering with phonons to obtain a common final temperature. Although this work is by no means an exhaustive inclusion of all the possible scattering processes, we present counterexamples for each of these assumptions through a system of electrons interacting variously with impurities, phonons, and internally (Coulomb). We discuss the origin of these violations as lying in the difference between relative and average (measurement) time dynamics.

We further show that the hot-electron model, which is used widely in the literature, is not to be taken at face value since a system where a temperature can be defined through the fluctuation-dissipation theorem will not have any dynamics at all. Rather, the dynamics is determined by the difference between two distribution functions-one each for the populations and one for the interactions.

These observations then raise the question: "How should one interpret nonequilibrium experiments, and what leads to the observed dynamics?" The answer, which we start to address here and in previous work [28], is that the dynamics of energy transfer between various subsystems controls the dynamics, but internal scattering within the subsystem can cause substantial modifications. For the situations that we study here, a return to equilibrium occurs only when a path (through the phonons) is provided for the excess energy in the electrons to dissipate. There are similarities between the energy dissipation characteristics and some of the equilibrium quantities since they share some of the underlying physical principles such as phase-space restrictions, leading to, e.g., the phonon window effect, although we should understand this effect as a phase-space restriction on the excess energy leaving the electrons rather than the singlequasiparticle lifetime.

When it comes to the interpretation of the obtained lifetimes, they should be taken as direct quantitative measures only if it can be shown that a single process is responsible for the decay and any internal scattering is absent, otherwise, the rate may contain many contributions, and it is not clear how to separate these. Nevertheless, progress may be made by varying the other external control parameters of the experiment and observing how the decay rates change as a function of said parameters; this approach is already used in the field but should be more strongly considered as a method of investigation using timeresolved techniques.

\section{ACKNOWLEDGMENTS}

We would like to acknowledge U. Bovensiepen, T. P. Devereaux, P. Kirchmann, and J. Sobota for helpful discussions. J. K. F. is supported by the U.S. Department of Energy, Office of Basic Energy Sciences, Materials Sciences and Engineering under Contract No. DE-FG0208ER46542 and also by the McDevitt bequest at Georgetown. Computational resources are provided by the National Energy Research Scientific Computing Center supported by the U.S. Department of Energy, Office of Science, under Contract No. DE-AC02$05 \mathrm{CH} 11231$.

[1] U. Schollwöck, The Density-Matrix Renormalization Group, Rev. Mod. Phys. 77, 259 (2005).

[2] J. K. Freericks, V. M. Turkowski, and V. Zlatić, Nonequilibrium Dynamical Mean-Field Theory, Phys. Rev. Lett. 97, 266408 (2006).

[3] H. Aoki, N. Tsuji, M. Eckstein, M. Kollar, T. Oka, and P. Werner, Nonequilibrium Dynamical Mean-Field Theory and Its Applications, Rev. Mod. Phys. 86, 779 (2014).

[4] M. Rigol, V. Dunjko, and M. Olshanii, Thermalization and Its Mechanism for Generic Isolated Quantum Systems, Nature (London) 452, 854 (2008).

[5] L. Stojchevska, I. Vaskivskyi, T. Mertelj, P. Kusar, D. Svetin, S. Brazovskii, and D. Mihailovic, Ultrafast Switching to a Stable Hidden Quantum State in an Electronic Crystal, Science 344, 177 (2014).

[6] P. B. Allen, Theory of Thermal Relaxation of Electrons in Metals, Phys. Rev. Lett. 59, 1460 (1987).

[7] V. Špička, B. Velický, and A. Kalvová, Long and Short Time Quantum Dynamics: I. Between Green's Functions and Transport Equations, Physica (Amsterdam) 29E, 154 (2005); Long and Short Time Quantum Dynamics: II. Kinetic Regime, Physica (Amsterdam) 29E, 175 (2005); Long and Short Time Quantum Dynamics: III. Transients, Physica (Amsterdam) 29E, 196 (2005).

[8] A. F. Kemper, M. Sentef, B. Moritz, C. C. Kao, Z. X. Shen, J. K. Freericks, and T. P. Devereaux, Mapping of Unoccupied States and Relevant Bosonic Modes via the TimeDependent Momentum Distribution, Phys. Rev. B 87, 235139 (2013).

[9] M. Sentef, A. F. Kemper, B. Moritz, J. K. Freericks, Z.-X. Shen, and T.P. Devereaux, Examining Electron-Boson Coupling Using Time-Resolved Spectroscopy, Phys. Rev. X 3, 041033 (2013).

[10] V. M. Galitskii and A. B. Migdal, Application of Quantum Field Theory Methods to the Many Body Problem, Sov. Phys. JETP 7, 96 (1958).

[11] G. D. Mahan, Many Particle Physics (Springer, New York, 2010).

[12] A. F. Kemper, M. A. Sentef, B. Moritz, J. K. Freericks, and T.P. Devereaux, Effect of Dynamical Spectral Weight Redistribution on Effective Interactions in Time-Resolved Spectroscopy, Phys. Rev. B 90, 075126 (2014).

[13] I. Gierz, S. Link, U. Starke, and A. Cavalleri, Non-Equilibrium Dirac Carrier Dynamics in Graphene 
Investigated with Time- and Angle-Resolved Photoemission Spectroscopy, Faraday Discuss. 171, 311 (2014).

[14] S.-L. Yang, J. A. Sobota, D. Leuenberger, Y. He, M. Hashimoto, D. H. Lu, H. Eisaki, P. S. Kirchmann, and Z.-X. Shen, Inequivalence of Single-Particle and Population Lifetimes in a Cuprate Superconductor, Phys. Rev. Lett. 114, 247001 (2015).

[15] A. Matthiessen and C. Vogt, Ueber den Einfluss der Temperatur auf die elektrische Leitungsfähigkeit der Legirungen, Ann. Phys. (Berlin) 198, 19 (1864).

[16] A.F. Kemper and J. K. Freericks, Relationship between Population Dynamics and the Self-Energy in Driven NonEquilibrium Systems, Entropy 18, 180 (2016).

[17] A. F. Kemper, H. R. Krishnamurthy, and J. K. Freericks, The Role of Average Time Dependence on the Relaxation of Excited Electron Populations in Nonequilibrium ManyBody Physics, Fortschr. Phys. 65, 1600042 (2017).

[18] B. Rethfeld, A. Kaiser, M. Vicanek, and G. Simon, Ultrafast Dynamics of Nonequilibrium Electrons in Metals under Femtosecond Laser Irradiation, Phys. Rev. B 65, 214303 (2002).

[19] V. V. Baranov and V. V. Kabanov, Theory of Electronic Relaxation in a Metal Excited by an Ultrashort Optical Pump, Phys. Rev. B 89, 125102 (2014).

[20] L. V. Keldysh, Diagram Technique for Nonequilibrium Processes, Sov. Phys. JETP 20, 1018 (1965).

[21] Y. Murakami, P. Werner, N. Tsuji, and H. Aoki, Interaction Quench in the Holstein Model: Thermalization Crossover from Electron- to Phonon-Dominated Relaxation, Phys. Rev. B 91, 045128 (2015).

[22] J. K. Freericks, K. Najafi, A. F. Kemper, and T. P. Devereaux, Nonequilibrium Sum Rules for the Holstein Model, in Proceedings of the Conference on Femtosecond
Electron Imaging and Spectroscopy, FEIS 2013, Key West (Academic Press, United Kingdom, 2015), p. 83.

[23] G. Stefanucci and R. van Leeuwen, Nonequilibrium ManyBody Theory of Quantum Systems: A Modern Introduction (Cambridge University Press, New York, 2013).

[24] R. Peierls, Zur Theorie des Diamagnetismus von Leitungselektronen, Z. Phys. 80, 763 (1933).

[25] J. K. Freericks and H. R. Krishnamurthy, Constant Matrix Element Approximation to Time-Resolved AngleResolved Photoemission Spectroscopy, Photonics 3, 58 (2016); J. K. Freericks, H. R. Krishnamurthy, and Th. Pruschke, Theoretical Description of Time-Resolved Photoemission Spectroscopy: Application to Pump-Probe Experiments, Phys. Rev. Lett. 102, 136401 (2009).

[26] R. Bertoncini and A.-P. Jauho, Gauge-Invariant Formulation of the Intracollisional Field Effect Including Collisional Broadening, Phys. Rev. B 44, 3655 (1991).

[27] V. Turkowski and J. K. Freericks, Nonequilibrium Dynamical Mean-Field Theory of Strongly Correlated Electrons, in Strongly Correlated Systems: Coherence and Entanglement, edited by J. M. P. Carmelo, J. M. B. Lopes dos Santos, V. Rocha Vieira, and P. D. Sacramento (World Scientific, Singapore, 2007), pp. 187-210.

[28] J. D. Rameau, S. Freutel, A. F. Kemper, M. A. Sentef, J. K. Freericks, I. Avigo, M. Ligges, L. Rettig, Y. Yoshida, H. Eisaki, J. Schneeloch, R. D. Zhong, Z. J. Xu, G. D. Gu, P. D. Johnson, and U. Bovensiepen, Energy Dissipation from a Correlated System Driven Out of Equilibrium, Nat. Commun. 7, 13761 (2016).

[29] M. Bauer, A. Marienfeld, and M. Aeschlimann, Hot Electron Lifetimes in Metals Probed by Time-Resolved Two-Photon Photoemission, Prog. Surf. Sci. 90, 319 (2015). 